\title{
La desvinculación del sector financiero con la economía real en el caso mexicano: una prueba de cointegración*
}

\author{
Óscar Javier de la Garza Garza** y Raúl Ángel Martínez Ibarra***
}

\begin{abstract}
RESUMEN
El objetivo principal de este artículo es analizar si en México el sector financiero está vinculado con la parte real de la economía. Para llevar a cabo este analisis, se hace uso de las técnicas de cointegración, que precisamente permiten probar la existencia de relaciones de largo plazo entre variables. La evidencia indica que hasta antes de la crisis bursátil mundial de octubre de 2008, la economía real estaba vinculada con el sector financiero, específicamente con el sector bursátil. Posteriormente a la crisis, se encuentra evidencia de que la economía real y los préstamos de la banca comercial mantienen una relación de largo plazo.
\end{abstract}

Palabras clave: sector financiero, economía real, series temporales, cointegración, desvinculación.

Clasificación JEL: G10, G22.

\begin{abstract}
This paper's main objective is to analyze whether the financial sector is linked to the real economy in Mexico. To accomplish this goal, it is contemplated using cointegration techniques that allow to precisely prove the existence of long-term relationships between variables. The evidence indicates that before the global stock market crash of October 2008, the real economy was linked to the financial sector, specifically with the securities industry. After the stock market crash, the evidence is that the real economy and loans from commercial banks maintain a long term relationship.
\end{abstract}

Keywords: financial sector, real economy, time series, cointegration, decoupling. JEL clasificación: G10, G22.

\footnotetext{
* Fecha de recepción: 15/05/2012. Fecha de aprobación: 21/02/2014.

** Profesor de cátedra de la Universidad Virtual del Instituto Tecnológico y de Estudios Superiores de Monterrrey. Correo electrónico: oscardelagarza78@yahoo.com.mx.

*** Profesor de cátedra de la Universidad Virtual del Instituto Tecnológico y de Estudios Superiores de Monterrrey. Correo electrónico: ramtzi@hotmail.com
} 


\section{INTRODUCCIÓN}

La reciente crisis financiera de 2008-2009 ha centrado nuevamente la atención de analistas y académicos sobre el rol del sector financiero y su vinculación con la economía real. Una de las tareas de quienes investigan el tema ha sido no sólo identificar si este sector incide positivamente en el crecimiento económico, sino también la relación que guarda con la parte real de la economía en el largo plazo. En ese marco, el objetivo principal de este trabajo es analizar si el sector financiero está vinculado con la economía real en México, para lo cual se hace uso de las técnicas de cointegración, las cuales precisamente permiten probar la existencia de relaciones de largo plazo entre variables que, en este caso, representan al sector real de la economía y al sector financiero.

La evidencia encontrada señala que hasta antes de la crisis bursátil mundial de octubre de 2008, la economía real, considerada a través del Indicador Global de la Actividad Económica (IGAE) o de la formación bruta de capital fijo en maquinaria y equipo, se hallaba vinculada con el sector financiero, específicamente con la bolsa, representado por el Índice de Precios y Cotizaciones (IPC). Con posterioridad a dicha crisis, existe evidencia de que el IGAE y los préstamos de la banca comercial mantienen una relación de largo plazo.

En la sección I se presenta la literatura relacionada con el tema, mientras que la evidencia empírica para el caso mexicano aparece en la II. Los datos y la metodología utilizados se encuentran en las secciones III y IV, mientras que los resultados y su interpretación se plantean en la sección v. Adicionalmente, se exponen las conclusiones pertinentes

\section{LITERATURA SOBRE LA RELACIÓN ENTRE FINANZAS Y ECONOMÍA REAL}

El rol del sector financiero en la economía ha sido ampliamente debatido en la literatura y, a partir de la reciente crisis financiera de 2008-2009, la cuestión ha llamado nuevamente la atención de analistas y académicos. En sus inicios, los trabajos académicos en torno al tema partían del modelo de crecimiento de Solow (1956), el cual sugería que, en el largo plazo, el crecimiento era conducido por el progreso tecnológico y no por la tasa de ahorros y, además, que las diferencias en el ingreso per cápita entre países reflejaban diferencias en las tasas de ahorro y de crecimiento de la población. Es importante notar que este modelo no considera en su configuración variables financieras. 
En contraste con la propuesta de Solow, John G. Gurley y E. S. Shaw (1955) plantearon, entre otras cosas, que para estudiar el crecimiento económico era necesario integrar al análisis del desarrollo en términos reales conceptos como la acumulación de la deuda y el crecimiento de intermediarios financieros no monetarios. En otras palabras, para estos autores, el sector financiero era un importante determinante del crecimiento económico.

Fue con las aportaciones de King y Levine (1993), Levine (1997), Levine y Zervos (1996) y Levine, Loayza y Beck (2000), que se logró conjuntar elementos en favor de la hipótesis de que el sector financiero impulsaba el crecimiento económico. En particular, estos trabajos indicaron que el crédito bancario al sector privado, la actividad del mercado de capitales y las características del sistema legal explicaban las diferencias en el crecimiento económico entre países, en el largo plazo. El argumento teórico es que los intermediarios financieros, al alentar la movilización de los ahorros, reducir los problemas de información asimétrica y proveer una mayor oportunidad para el risk spreading ${ }^{1} \mathrm{y}$ el risk pooling ${ }^{2}$, generan, a nivel agregado, mayor ahorro y una asignación más eficiente de recursos que afecta positivamente las tasas de acumulación del capital y de innovación tecnológica (Favara, 2003).

Otros hallazgos similares fueron los de Arestis, Demetriades y Luintel (2001), quienes aportan evidencia para afirmar que la banca y los mercados accionarios promueven el crecimiento económico real. Guiso, Sapienza, y Zingales (2004) mencionan que un desarrollo apropiado del sistema financiero abre la posibilidad de que los individuos inicien nuevos negocios, lo que significa que se fomenta el emprendimiento (entrepreneur) ${ }^{3}$. Shen y Lee (2006) muestran que el desarrollo del mercado accionario contribuye al florecimiento de la economía real. Por otra parte, Favara (2003), por ejemplo, encuentra que el nivel de desarrollo financiero tiene efectos ambiguos sobre el crecimiento económico.

No obstante, a partir de los noventa, se desarrolló una línea de investigación que sostiene que ni el desarrollo de los mercados financieros, ni la forma en

${ }^{1}$ El riesgo de que las inversiones de compensación (long-short positions) en una estrategia de cobertura experimenten cambios en los precios en direcciones completamente opuestas entre sí. Esta correlación imperfecta entre las dos inversiones crea el potencial de ganancias o pérdidas en exceso en una estrategia de cobertura, aumentando así el riesgo para la posición

${ }^{2}$ El concepto básico de riesgo compartido es distribuir o reducir el riesgo de que una sola entidad absorba una perdida en caso de un evento inesperado. Por lo tanto, el grupo de riesgo reduce la exposición a pérdidas financieras mediante la distribución del riesgo entre sus miembros.

${ }^{3}$ Que se define como la capacidad de iniciar nuevas empresas o nuevos proyectos. 
que éstos se organizan (bancario o de capitales), favorecen el crecimiento de la economía real, por lo que Menkhoff y Tolksdorf (2001) desarrollaron la teoría de la desvinculación o decoupling. En la literatura encontramos una serie de estudios que buscan probar dicha hipótesis, utilizando principalmente la técnica de cointegración. Cheung y Ng (1998) encuentran evidencia a favor de la relación de largo plazo entre actividad agregada real -medida a través de precio del petróleo-, consumo, oferta monetaria y producción, y los índices del mercado accionario. Esto, en los casos de Canadá, Italia, Japón y Estados Unidos (EU) durante el periodo 1957-1992.

Ansotegui y Esteban (1997), en un estudio sobre España, utilizan el Índice de la Bolsa de Madrid y para los fundamentos de la economía incluyen la producción industrial, la inflación y la tasa de interés desde 1980 hasta 1992. En sus resultados, estos investigadores reportan la existencia de relaciones de largo plazo entre el mercado de capitales y las variables macroeconómicas. Por su parte, Kwon y Shin (1999) analizan si la actividad económica de Corea del Sur puede explicar los rendimientos del mercado de acciones utilizando una prueba de cointegración, a partir de un modelo de corrección de errores, para el cual usan el índice de la producción industrial, el tipo de cambio, la balanza comercial y la oferta monetaria como variables macroeconómicas; los resultados constatan que el mercado accionario coreano refleja las variables macroeconómicas en los precios de los índices bursátiles durante el periodo comprendido entre 1980 y 1982.

Asimismo, Nasseh y Strauss (2000), utilizando análisis de cointegración, señalan que los precios de las acciones están significativamente relacionados con la producción industrial y la tasa de interés de largo plazo en Francia, Alemania, Italia, Holanda, Suiza y el Reino Unido durante el periodo 1962-1995. McMillan (2001) realiza un análisis similar al de Nasseh y Strauss aplicado al mercado accionario de EU; en este caso, las variables que utiliza para el sector financiero son el índice Standard \& Poor's 500 y el Dow Jones Industrial Average, y para la actividad económica, la producción industrial, la inflación, la oferta monetaria y la tasa de interés. El propio McMillan (2005) llevo a cabo otro estudio semejante utilizando datos de 1970 a 2000, en el que encontró que los precios muestran una relación positiva con la producción industrial y una negativa con la tasa de interés.

Por su parte, Maysami, Howe y Hamzah (2004) reportan una relación de largo plazo entre producción industrial, precios, tipo de cambio, oferta monetaria y tasas de interés, como variables macroeconómicas, y los índices bursátiles de 
Singapur de 1989 a 2001, utilizando el método de cointegración. Del mismo modo, Humpe y Macmillan (2007) analizan si hay variables macroeconómicas que pueden explicar movimientos en el mercado de valores y revisan si, en el caso de EU y Japón, la producción industrial, el índice de precios, la oferta monetaria, la tasa de interés y el índice bursátil guardan una relación de largo plazo. Estos autores hallan que los precios de las acciones son influenciados positivamente por la producción industrial y negativamente por la inflación y la tasa de interés en el caso estadounidense. Para el japonés, concluyen que el precio de las acciones se ve influenciado positivamente por la producción industrial y negativamente por la oferta monetaria.

Cagli, Halac y Taskin (2010) investigan la relación entre variables macroeconómicas y los mercados bursátiles en Turquía durante 1998-2008, utilizando el producto interno bruto (PIB), el precio del petróleo y la producción industrial, así como el Istanbul Stock Exchange National 100, y encuentran una relación de largo plazo, incluso en un estudio anterior sobre la economía turca. Karamustafa y Kucukkale (2003), empleando como variables macroeconómicas la oferta monetaria, el tipo de cambio, la balanza comercial y el índice de la producción industrial, y también el Istanbul Stock Exchange National 100, encuentran los mismos resultados.

\section{EVIDENCIA EMPÍRICA EN EL CASO MEXICANO}

En el caso de México, Morales y Rendón (1997) muestran que el comportamiento accionario, medido a través del IPC de la Bolsa Mexicana de Valores, puede ser explicado por el PIB nominal y la tasa de los Certificados de la Tesorería (Cetes) a 28 días, utilizando pruebas de cointegración. En su estudio, señalan que sí existe relación entre el comportamiento económico y el movimiento bursátil, en el lapso de 1989 a 1996.

Por otro lado, Taboada y Sámano (2003) llevaron a cabo una investigación utilizando la formación bruta de capital fijo en maquinaria y equipo como la variable de medición indirecta de la economía real. Para representar el sistema financiero mexicano emplean el IPC y los préstamos bancarios al sector industrial durante el periodo de 1993 a 2001. Utilizando el análisis de cointegración encontraron que existe una desvinculación entre el sistema financiero mexicano y la economía real. Estos autores muestran que el sistema financiero tiene una dinámica independiente que no permite establecer la presencia de relaciones de largo plazo entre las variables. 
Por su parte, Zavaleta y Urbina (2011) encuentran una relación entre el desempeño del mercado accionario y el crecimiento de la economía real en México. Como variable de medición indirecta del sector financiero utilizan el IPC y para la economía real, el IGAE, de 1993 a 2010. Cabe señalar que ninguno de estos estudios toma en cuenta la posibilidad de cambios estructurales. Según Domingo y Tonella (2000), los cambios estructurales pueden modificar las características esenciales de las variables, desde grados pequeños y casi imperceptibles hasta alteraciones totales, es decir, los cambios estructurales pueden repercutir de tal manera que las relaciones en las series analizadas pueden aparecer y desaparecer a lo largo del tiempo.

\section{DATOS Y VARIABLES}

Las variables usadas en esta investigación para la medición indirecta de la dinámica del sistema financiero fueron los créditos otorgados por la banca comercial al sector industrial y el IPC, mientras que para la actividad económica real, fueron el índice de actividad industrial, el IGAE y la formación bruta de capital fijo en maquinaria y equipo. Los datos utilizados son mensuales de enero de 1996 a mayo del 2013 y fueron obtenidos del Banco de Información Económica del Instituto Nacional de Estadística y Geografía (INEGI, s.f.a).

Se decidió caracterizar a la economía real mediante la formación bruta de capital fijo de maquinaria y equipo (grafica 1). Al respecto, hay que señalar

Gráfica 1. Formación bruta de capital fijo en maquinaria y equipo

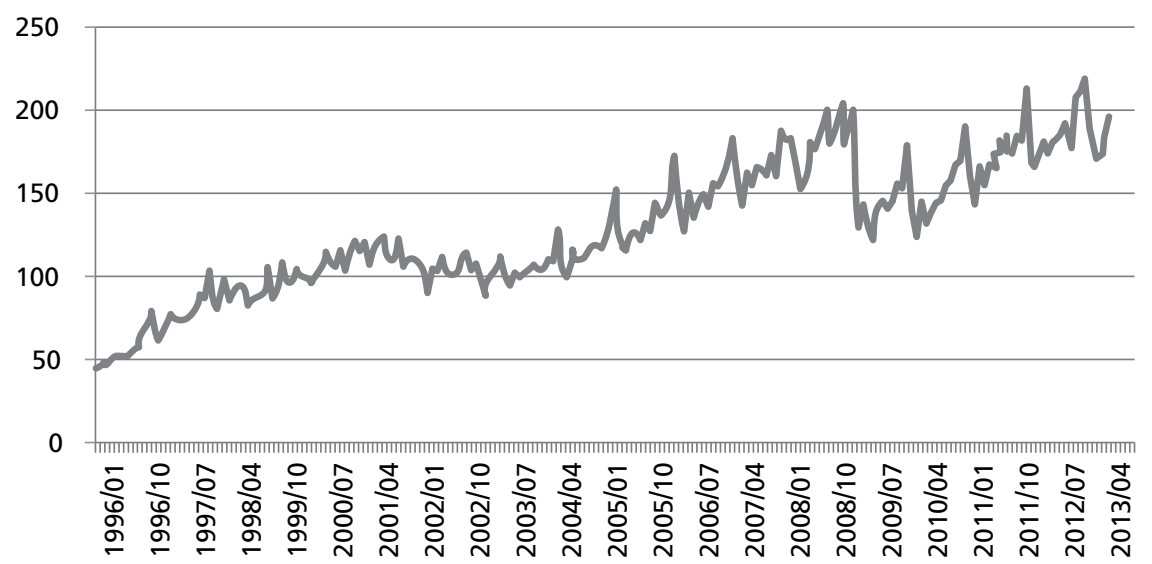

Fuente: Elaboración propia con información de INEGI (s.f.a). Unidad de medida: Índice base enero de $2003=100$. 
que si se tomara el PIB como variable indirecta, sería redundante, en el sentido de que contempla al propio sistema financiero, además de que su periodicidad es trimestral. La formación bruta de capital fijo en maquinaria y equipo evita contabilizar la inversión en construcción, para no sesgar los resultados, ya que una parte de esta inversión contiene lo que se destina al sector vivienda, que no es propiamente inversión productiva. Además, esta variable refleja el crecimiento real de la economía, ya que la demanda de maquinaria y equipo significa en buena medida que las empresas están utilizando intensivamente su capacidad instalada, aunque, como se sabe, pueden estar realizando adquisiciones para reposición.

Adicionalmente, se utiliza el IGAE, indicador que muestra la evolución de la actividad económica del país (grafica 2). Para su elaboración se utiliza el esquema conceptual y metodológico de la contabilidad nacional, el mismo que sigue el cálculo del PIB trimestral, Es importante destacar que la información básica que incorpora este indicador es muy preliminar, está sujeta a cambios y no incluye todas las actividades económicas, como sucede con el PIB trimestral, pero sí contiene información con respecto al sistema financiero. Por ello, sus resultados pueden diferir de los del PIB trimestral y debe considerársele como un indicador de la tendencia o dirección de la actividad económica en el país en el corto plazo. Sin embargo, como ya se mencionó, la formación bruta de capital fijo en maquinaria y equipo funcionaría mejor como una variable para medir indirectamente la actividad económica real y la inclusión del IGAE tiene la finalidad de comparar el presente estudio con otros trabajos sobre caso mexicano.

Gráfica 2. Indicador Global de la Actividad Económica

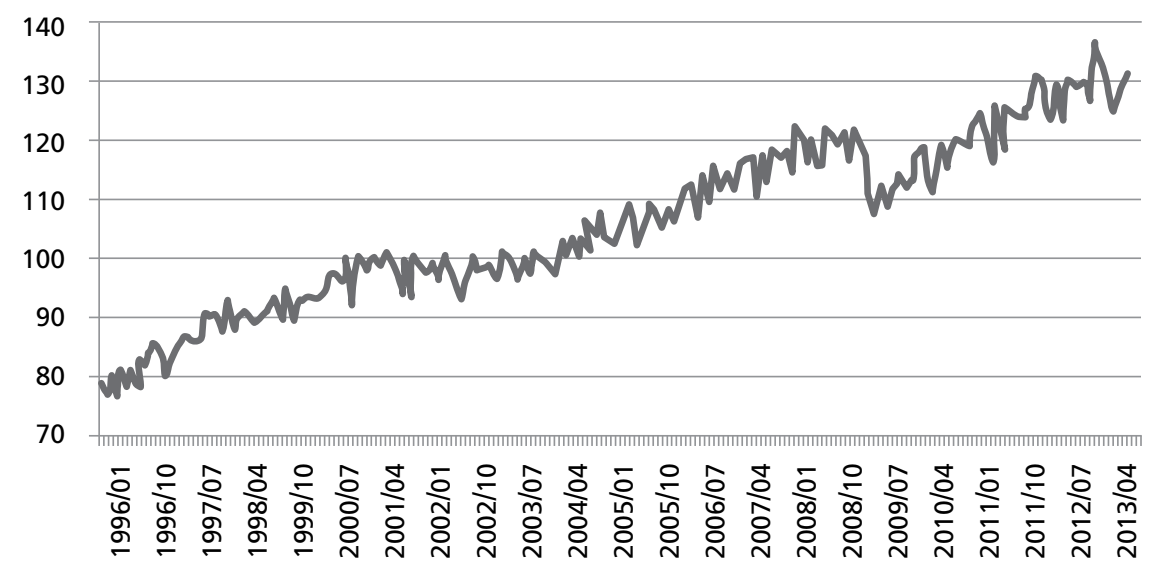

Fuente: INEGI (s.f.a). Unidad de medida: Índice base enero de $2003=100$. 
Por otra parte, también se utiliza como variable para la medición indirecta de la economía real el índice de actividad industrial, que expresa el volumen físico para 321 de 374 clases de actividad económica del Sistema de Clasificación Industrial de América del Norte (SCIAN) (INEGI, s.f.c). La principal fuente de información es la Encuesta Industrial Mensual del Instituto Nacional de Estadística y Geografía (INEGI, s.f.b) (grafica 3). Es importante destacar que estos datos son compatibles con los cálculos publicados sobre el PIB. La razón de incluir esta variable, además de su alta correlación con la actividad económica, es la posibilidad de realizar comparaciones, ya que los estudios a nivel internacional sobre este tema utilizan principalmente este tipo de índices para medir la actividad económica real.

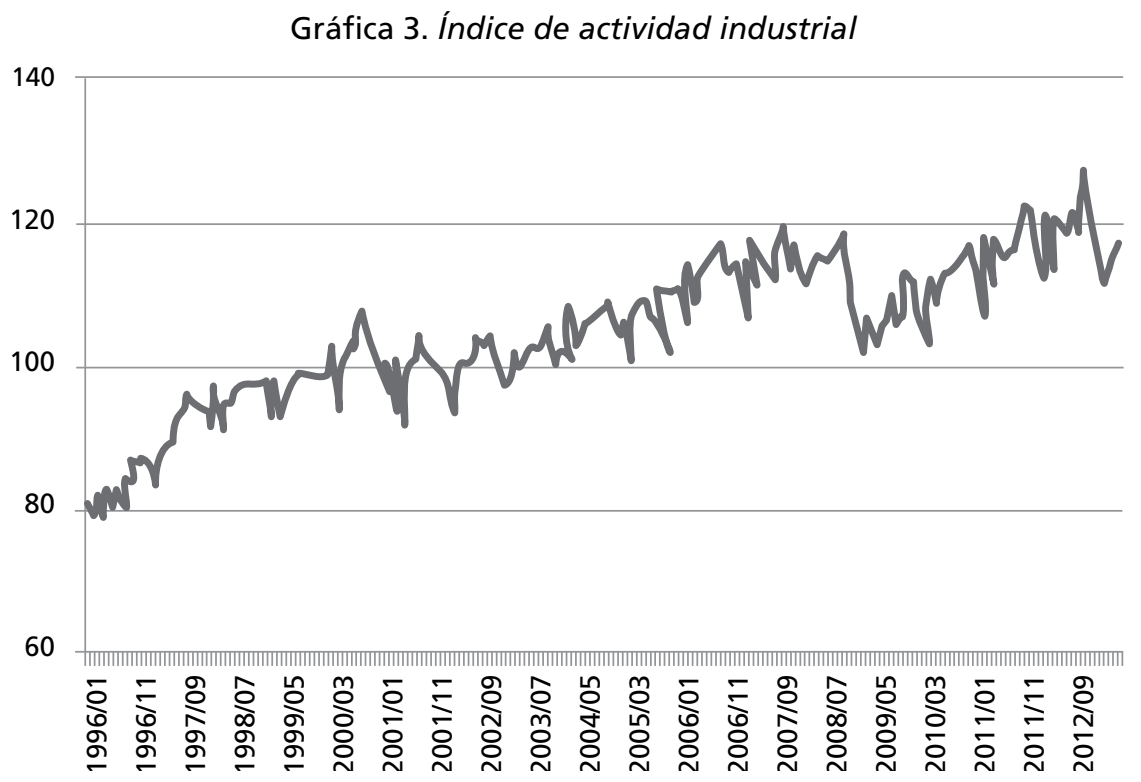

Fuente: INEGI (s.f.a). Unidad de medida: Índice base enero de $2003=100$.

Por el lado del sistema financiero, la mayoría de los trabajos toman el IPC como variable para medir indirectamente este sector en el caso mexicano $\mathrm{y}$, asimismo, este tipo de índices accionarios son los que se utilizan principalmente en estudios a nivel internacional (grafica 4). El IPC es un indicador de la evolución del mercado accionario que se calcula en función de las variaciones de una selección de acciones, llamada muestra, balanceada, ponderada y representativa de todas las acciones cotizadas en la Bolsa Mexicana de Valores. 


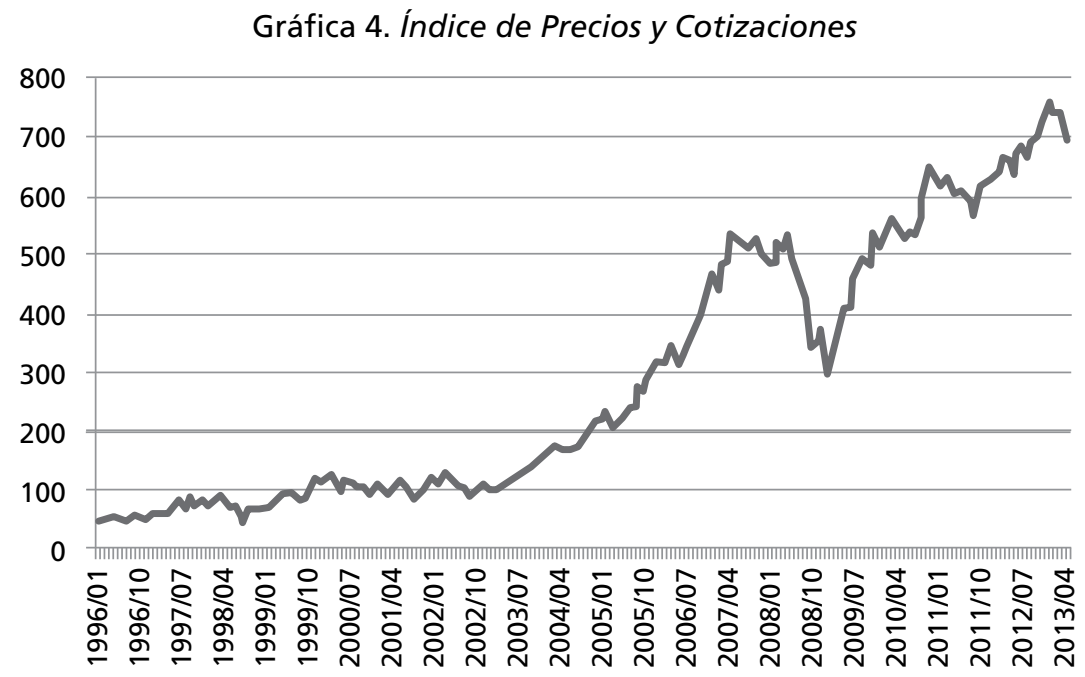

Fuente: INEGI (s.f.a). Unidad de medida: Índice base enero de $2003=100$.

Gráfica 5. Préstamos de la banca comercial

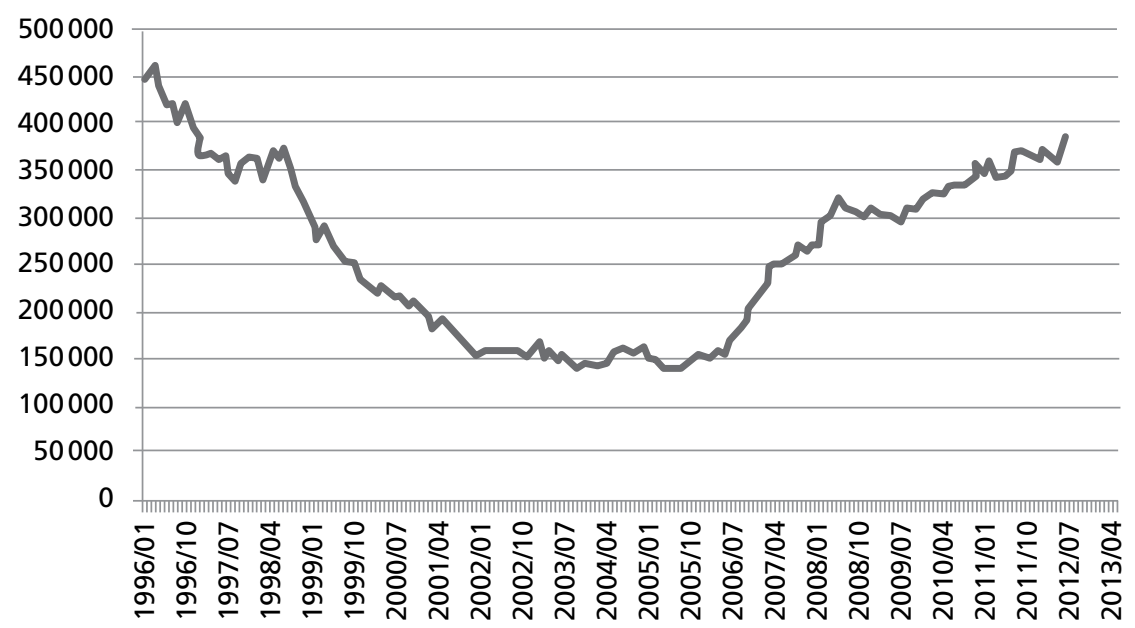

Fuente: INEGI (s.f.a). Unidad de medida: Saldos reales en millones de pesos.

Por otra parte, una variable que refleja la actividad del sector financiero y puede respaldar o, en su caso, refutar los resultados obtenidos mediante el análisis del sector bursátil, son los préstamos bancarios al sector industrial (gráfica 5). Tales créditos indican de manera más tangible si el sistema financiero está cumpliendo con su función de intermediación entre ahorradores y empresas o em- 
prendedores. Los préstamos bancarios están directamente relacionados con la formación bruta de capital fijo en maquinaria y equipo requeridos por el sector industrial para producir, ya que su adquisición requiere de financiamiento. Hay que recordar que éste proviene de tres fuentes: recursos propios, bolsa de valores y préstamos bancarios. En este sentido, para que los resultados fueran más robustos, se definió como sector financiero a la bolsa de valores y al sector bancario, en la medida en que se pretende analizar la posible desvinculación del sistema financiero con la economía real.

\section{METODOLOGÍA: COINTEGRACIÓN DE SERIES TEMPORALES}

El uso de técnicas de series temporales permite probar la existencia de relaciones de largo plazo entre variables, ${ }^{4}$ así como establecer la causalidad entre dos o más de ellas. Desde el punto de vista econométrico, dos o más series temporales que son no estacionarias ${ }^{5}$ de orden $I(1)$ están cointegradas si existe una combinación lineal de esas series que sea estacionaria de orden $I(0)$. Cointegración ${ }^{6}$ quiere decir que existe una relación a largo plazo entre las variables, de modo que cuando dos variables están cointegradas significa que aunque crezcan en el tiempo el error entre ambas series no crece. Kwon y Shin (1999) indican que si las variables macroeconómicas se reflejan en los mercados financieros, entonces deberían estar cointegradas. ${ }^{7}$ Si no lo están, podríamos concluir que el mercado accionario no proporciona señales sobre la actividad económica.

El análisis de cointegración, sugerido por Engle y Granger (1987) y generalizado al caso multivariado por Johansen (1991), se ocupa de la naturaleza del largo plazo; en particular, permite determinar si series integradas del mismo orden pueden combinarse para una serie estacionaria. Si ése es el caso se dice que las series están cointegradas y que necesariamente comparten una tendencia estocástica. En otras palabras, si las series en cuestión son integradas de orden 1,

\footnotetext{
${ }^{4}$ Las estimaciones de regresiones con variables no estacionarias son espurias, salvo que estas estén cointegradas.

${ }^{5}$ Son las variables que tienen una tendencia temporal definida, es decir, su valor medio cambia a lo largo del tiempo.

${ }^{6}$ Las relaciones no cointegradas no siempre son espurias. Por ello hay que ser prudentes ante los resultados de no cointegración, pues no deben llevarnos a afirmar de forma terminante que la relación entre variables es espuria. La aceptación de la cointegración no es condición ni necesaria ni suficiente para afirmar que entre las variables existe una relación no espuria.

${ }^{7} \mathrm{Si}$ esto se da, entonces se existe racionalidad, lo que significa que el mercado financiero está relacionado y dirigido por sus fundamentos en el largo plazo.
} 
entonces tendrán una raíz unitaria, de tal forma que hay una tendencia estocástica que no las hará converger hacia ningún trayecto determinístico en particular, por lo que la cointegración implica que las series tendrán una tendencia similar en el largo plazo.

El análisis parte del modelo de vectores autorregresivos (VAR) para el vector $X_{t}$ de orden $(k \times 1)$ formado por variables integradas de orden 1:

$$
\Delta X_{t}=\mu+\sum_{i=1}^{p} A_{i} \Delta X_{t-\mathrm{i}}+\varepsilon_{t}
$$

donde $\Delta X_{t}$ y $\Delta X_{t-1}$ son vectores de orden $(k \times 1)$ y $A_{i}$ son matrices de orden $(k \times 1)$ de parámetros desconocidos.

Este modelo es reformulado como un modelo vectorial de corrección de errores:

$$
\Delta X_{t}=\mu+\sum_{i=1}^{p-1} \Gamma_{i} \Delta X_{t-i}+\Pi X_{t-p}+\varepsilon_{t}
$$

donde $\Gamma_{i}=-I+A_{i}+\cdots+A_{i}, \Pi=-\left(I-A_{i}-\cdots-A_{p}\right)$ e $I$ es una matriz unitaria de orden $k$. El análisis de cointegración se concentra en el rango de la matriz $\Pi$, ya que son $k$ variables que constituyen el vector $X_{t}$, el rango máximo de $\Pi$ es $k$. Ahora bien, si $\rho(\Pi)=0, \Pi$ es una matriz nula y el modelo sería un modelo de VAR en primeras diferencias $\rho(\Pi)=k$, el proceso es estacionario. En una situación intermedia con $\rho(\Pi)=1$, habría un vector cointegrador y la expresión $\Pi X_{t-p}$ sería estacionaria. En el caso en el que $\rho(\Pi)=r$, con $1<r<k$ habría múltiples vectores cointegradores y se dice que $\Pi$ tendría rango igual a $r$. En este caso, existirían las matrices $\alpha$ y $\beta$ cada una con rango $r$, tal que $\Pi=\alpha \beta$, de modo que el mecanismo de corrección de errores es estacionario.

El número de vectores cointegradores diferentes puede obtenerse evaluando la significación estadística de las raíces características de $\Pi$, ya que el rango de una matriz es igual al número de raíces características que son diferentes de cero. En la práctica, se obtienen estimaciones por máxima verosimilitud de $\Pi$ y de sus raíces características. La prueba del número de raíces características que son significativamente iguales a cero, se realiza mediante el siguiente estadístico de prueba: 


$$
\lambda_{\text {traza }}(r)=-T \sum_{i=r+1}^{k} \operatorname{In}\left(1-\lambda_{i}\right)
$$

donde $\hat{\lambda}_{i}$ es la $i$-ésima raíz característica y $T$ es el número de observaciones. Este estadístico evalúa la hipótesis nula de que el número de vectores cointegradores es menor o igual a $r$ contra una hipótesis alternativa general. Los valores críticos son proporcionados por Johansen (1995).

Sin embargo, los cambios estructurales pueden repercutir de tal manera que aparezcan, desaparezcan o cambien las relaciones en las series analizadas. Por consiguiente, cuando éstas presentan cambios estructurales que no se toman en cuenta, las pruebas tradicionales de cointegración tienden a no rechazar la hipótesis nula (no cointegración), incluso a pesar de que sí se cointegren.

En la práctica, no siempre es posible saber con exactitud cuándo han ocurrido cambios estructurales en la serie analizada. Las pruebas propuestas por Zivot y Andrews (1992) permiten que la función de tendencia de la serie considere cambios que ocurren en un periodo que no se conoce previamente. Dichos autores sugieren que los cambios estructurales se consideren endógenos y proponen un algoritmo para determinar los puntos de ruptura desconocidos. La selección del periodo de ruptura se determina con el valor más negativo de la $t$ en una prueba de raíz unitaria del tipo de Dickey-Fuller aumentada, difiriendo de ésta en los valores críticos. Si se supone que hay $q-1$ rupturas, la muestra total estaría formada por $q$ subperiodos para cada uno de los cuales se podrían efectuar las pruebas convencionales.

\section{Resultados}

Primero, se presenta la matriz de correlaciones en el cuadro 1. Las correlaciones no condicionales sugieren que las variables que representan a la economía real y al sector financiero podrían estar integradas en el caso específico del sector bursátil. Sin embargo, en el caso de los créditos de la banca comercial, el signo es el opuesto al esperado. ${ }^{8}$ De acuerdo con Kasa (1992), la información proporcionada por los coeficientes de correlación no es suficiente para determinar si existe o no integración entre las variables.

${ }^{8}$ La matriz de correlación del periodo febrero de 2009-mayo de 2013, en el caso de los créditos de la banca comercial, sí tiene el signo esperado, positivo. 
Cuadro 1. Matriz de correlaciones

\begin{tabular}{|c|c|c|c|c|c|}
\hline & IGAE & $\begin{array}{l}\text { Actividad } \\
\text { industrial }\end{array}$ & $\begin{array}{c}\text { Formación } \\
\text { bruta de } \\
\text { capital }\end{array}$ & IPC & $\begin{array}{l}\text { Créditos } \\
\text { de la banca } \\
\text { comercial }\end{array}$ \\
\hline IGAE & 1.0000 & & & & \\
\hline $\begin{array}{l}\text { Actividad } \\
\text { industrial }\end{array}$ & 0.9571 & 1.0000 & & & \\
\hline $\begin{array}{l}\text { Formación bruta } \\
\text { de capital }\end{array}$ & 0.9627 & 0.9339 & 1.0000 & & \\
\hline IPC & 0.9319 & 0.8276 & 0.8797 & 1.0000 & \\
\hline $\begin{array}{l}\text { Créditos de la } \\
\text { banca comercial }\end{array}$ & -0.0506 & -0.2303 & -0.0668 & 0.2164 & 1.000 \\
\hline
\end{tabular}

En el cuadro 2 se presentan las pruebas de raíces unitarias de Dickey y Fuller (1979) y Phillips y Perron (1988) para los niveles y en primeras diferencias. Los resultados muestran que las variables asociadas al sector financiero (créditos otorgados por la banca comercial al sector industrial e IPC) y dos de las tres que representan a la economía real (IGAE y formación bruta de capital fijo en maquinaria y equipo) son no estacionarias. En el caso de la variable de actividad industrial, las pruebas en niveles se contraponen.

Cuadro 2. Pruebas de raíces unitarias en niveles

\begin{tabular}{lclc}
\hline \multicolumn{2}{c}{ Prueba de Dickey-Fuller } & \multicolumn{2}{c}{ Prueba de Phillips-Perron } \\
\hline IGAE & -1.751 & IGAE & -1.020 \\
& $(0.4051)$ & & $(0.7459)$ \\
\hline Actividad industrial & $-2.916^{*}$ & Actividad industrial & -2.364 \\
& $(0.0435)$ & & $(0.1522)$ \\
\hline Formación bruta & -2.703 & Formación & -2.083 \\
de capital & $(0.0735)$ & bruta de capital & $(0.2514)$ \\
IPC & 0.486 & IPC & 0.270 \\
& $(0.9844)$ & & $(0.9759)$ \\
Créditos de la banca & -1.695 & Créditos de la banca & -1.606 \\
comercial & $(0.4338)$ & comercial & $(0.4806)$ \\
\hline
\end{tabular}

* Significación al 5\%. 
Cuadro 3. Pruebas de raíces unitarias en primeras diferencias

\begin{tabular}{|c|c|c|c|}
\hline \multicolumn{2}{|c|}{ Prueba de Dickey-Fuller } & \multicolumn{2}{|c|}{ Prueba de Phillips-Perron } \\
\hline$\Delta \mathrm{IGAE}$ & $\begin{array}{r}-22.805^{*} \\
(0.0000)\end{array}$ & $\Delta \mathrm{IGAE}$ & $\begin{array}{r}-29.824^{*} \\
(0.0000)\end{array}$ \\
\hline $\begin{array}{c}\Delta \text { Actividad } \\
\text { industrial }\end{array}$ & $\begin{array}{r}-26.202^{*} \\
(0.0000)\end{array}$ & $\begin{array}{r}\Delta \text { Actividad } \\
\text { Industrial }\end{array}$ & $\begin{array}{r}-26.823^{*} \\
(0.0000)\end{array}$ \\
\hline $\begin{array}{l}\Delta \text { Formación bruta } \\
\text { de capital }\end{array}$ & $\begin{array}{r}-19.601 * \\
(0.0000)\end{array}$ & $\begin{array}{l}\Delta \text { Formación bruta } \\
\text { de capital }\end{array}$ & $\begin{array}{r}-22.278^{*} \\
(0.0000)\end{array}$ \\
\hline$\Delta \mathrm{IPC}$ & $\begin{array}{r}-13.355^{*} \\
(0.0000)\end{array}$ & $\Delta \mathrm{IPC}$ & $\begin{array}{r}-13.497^{*} \\
(0.0000)\end{array}$ \\
\hline $\begin{array}{l}\Delta \text { Créditos de la } \\
\text { banca comercial }\end{array}$ & $\begin{array}{r}-11.596^{*} \\
(0.0000)\end{array}$ & $\begin{array}{l}\Delta \text { Créditos de la } \\
\text { banca comercial }\end{array}$ & $\begin{array}{r}-11.918^{*} \\
(0.0000)\end{array}$ \\
\hline
\end{tabular}

* Significación al $1 \%$.

Asimismo, ambas pruebas en primeras diferencias para las variables antes mencionadas, nos indican que son estacionarias, lo que significa que son integradas de orden 1, I(1), lo que se puede ver en el cuadro 3. Sin embargo, estos resultados deben de tomarse con reserva, pues no consideran la presencia de cambios estructurales. La prueba de raíces unitarias propuesta por Zivot y Andrews (1992) sí permite tomar en cuenta posibles cambios estructurales en la función de tendencia de la serie. Los resultados se presentan en el cuadro 4.

Según los resultados de la prueba de Zivot y Andrews, el IGAE, el índice de actividad industrial y la formación bruta de capital fijo en maquinaria y equipo $^{9}$ presentan cambios estructurales en la función de tendencia, mientras que para las variables asociadas al sector financiero (los créditos otorgados por la banca comercial al sector industrial y el IPC) no hay evidencia estadística de cambios estructurales. Para tomar en cuenta la presencia de cambios estructurales en la función de la tendencia de las series asociadas a la economía real durante el periodo total que cubre la muestra de las variables analizadas (enero de 1996 a mayo del 2013), se realizaron pruebas de la traza para los siguientes subperiodos: enero de 1996 a octubre de 2008 y febrero de 2009 mayo de 2013.

La crisis bursátil mundial de octubre de 2008, que formó parte de la crisis financiera de 2008-2009, coincide con la división de la muestra en los dos

\footnotetext{
${ }^{9}$ No hay evidencia de cambio en la tasa de crecimiento de la serie.
} 
Cuadro 4. Prueba de raíz unitaria en presencia de cambio estructural (Zivot y Andrews)

\begin{tabular}{|lcc|}
\hline \multicolumn{1}{|c}{ Variable } & Modelo A & Modelo B \\
\hline IGAE & $\begin{array}{c}-5.547^{*} \\
\left(\frac{\text { noviembre }}{2008}\right)^{\mathrm{a}}\end{array}$ & $-5.046^{*}$ \\
\hline Actividad industrial & $-6.580^{*}$ & $\left(\frac{\text { septiembre }}{1998}\right)^{\mathrm{a}}$ \\
& $\left(\frac{\text { noviembre }}{2008}\right)^{\mathrm{a}}$ & $-5.051^{*}$ \\
Formación bruta de & $-4.911^{* *}$ & $\left(\frac{\text { julio }}{2006}\right)^{\mathrm{a}}$ \\
capital & $\left(\frac{\text { enero }}{2009}\right)^{\mathrm{a}}$ & -3.763 \\
\hline IPC & -3.269 & -3.570 \\
Créditos de la banca & -3.848 & -3.198 \\
comercial & -5.43 & -4.93 \\
Valor crítico al 1\% & -4.80 & -4.42 \\
\hline Valor crítico al $5 \%$ & & \\
\hline
\end{tabular}

* Significación al $1 \%$.

** Significación al $5 \%$.

Nota: $H_{0}$ : proceso con una raíz unitaria y deriva (drift) diferente de cero, excluyendo algún cambio estructural. $H_{A}$ : proceso estacionario en tendencia con un periodo de ruptura en la tendencia que ocurre en un momento desconocido.

a y b corresponden, respectivamente, a las hipótesis de cambio en el intercepto de la tendencia de la serie y cambio en el intercepto como en la pendiente de la tendencia (cambio en la tasa de crecimiento de la serie).

a Fecha de ruptura estructural, según la prueba.

Los valores críticos se toman de las tablas de Zivot y Andrews (1992).

subperiodos señalados. Dicha división corresponde a los resultados obtenidos en el cuadro 4. Cabe mencionar que esta crisis bursátil consistió en una caída histórica de las cotizaciones de prácticamente todas las bolsas del mundo, comparable con el crack financiero de 1929, que dio origen a la Gran Depresión.

Para seguir la técnica propuesta por Johansen (1988; 1991; 1992), se realizaron pruebas para los subperiodos de enero de 1996 a octubre de 2008 y de febrero de 2009 a mayo de 2013. Es de hacer notar que se cuenta con 154 observaciones y 52 observaciones, respectivamente, ya que para el uso de series temporales se recomienda como mínimo cincuenta observaciones. Los resultados se presentan en el cuadro 5 . 
122 ECONOMÍA: TEORÍA Y PRÁCTICA • Nueva Época, número 41, julio-diciembre 2014

\section{Cuadro 5. Pruebas de cointegración (primera parte)}

\begin{tabular}{|c|c|c|c|c|c|}
\hline \multicolumn{6}{|c|}{ Pruebas de la traza ( $\lambda_{\text {traza }}$ ) y el valor característico mínimo ( $\lambda_{\text {mínimo }}$ ) } \\
\hline \multicolumn{3}{|c|}{$\begin{array}{l}\text { Enero de } 1996 \text { a octubre de } 2008 \\
\text { IGAE e IPC }\end{array}$} & \multicolumn{3}{|c|}{$\begin{array}{l}\text { Febrero de } 2009 \text { a diciembre de } 2013 \\
\text { IGAE e IPC }\end{array}$} \\
\hline$H_{0}$ & $\lambda_{\text {traza }}$ & $\lambda_{\text {mínimo }}$ & $H_{0}$ & $\lambda_{\text {traza }}$ & $\lambda_{\text {mínimo }}$ \\
\hline$r=0$ & $8.3909 *$ & 15.41 & $r=0$ & 25.5350 & 15.41 \\
\hline$r=1$ & 0.3822 & 3.76 & $r=1$ & 5.0386 & 3.76 \\
\hline \multicolumn{3}{|c|}{$\begin{array}{l}\text { Enero de } 1996 \text { a octubre de } 2008 \\
\text { IGAE y préstamos de la banca comercial }\end{array}$} & \multicolumn{3}{|c|}{$\begin{array}{l}\text { Febrero de } 2009 \text { a mayo de } 2013 \\
\text { IGAE y préstamos de la banca comercial }\end{array}$} \\
\hline$H_{0}$ & $\lambda_{\text {traza }}$ & $\lambda_{\text {mínimo }}$ & $H_{0}$ & $\lambda_{\text {traza }}$ & $\lambda_{\text {mínimo }}$ \\
\hline$r=0$ & 29.2168 & 15.41 & $r=0$ & 16.9877 & 15.41 \\
\hline$r=1$ & $0.4025^{*}$ & 3.76 & $r=1$ & $0.0370 *$ & 3.76 \\
\hline
\end{tabular}

* Significación al $5 \%$.

Cuadro 6. Pruebas de cointegración (segunda parte)

Pruebas de la traza ( $\left.\lambda_{\text {traza }}\right)$ y el valor característico mínimo $\left(\lambda_{\text {mínimo }}\right)$

\begin{tabular}{|c|c|c|c|c|c|}
\hline \multicolumn{3}{|c|}{$\begin{array}{l}\text { Enero de } 1996 \text { a octubre de } 2008 \\
\text { Formación bruta de capital e IPC }\end{array}$} & \multicolumn{3}{|c|}{$\begin{array}{l}\text { Febrero de } 2009 \text { a mayo de } 2013 \\
\text { Formación bruta de capital e IPC }\end{array}$} \\
\hline$H_{0}$ & $\lambda_{\text {traza }}$ & $\lambda_{\text {mínimo }}$ & $H_{0}$ & $\lambda_{\text {traza }}$ & $\lambda_{\text {mínimo }}$ \\
\hline$r=0$ & 15.6595 & 15.41 & $r=0$ & 29.7068 & 15.41 \\
\hline$r=1$ & $0.5276^{*}$ & 3.76 & $r=1$ & 4.4323 & 3.76 \\
\hline \multicolumn{3}{|c|}{$\begin{array}{l}\text { Enero de } 1996 \text { a octubre del } 2008 \\
\text { Formación bruta de capital y préstamos de la } \\
\text { banca comercial }\end{array}$} & \multicolumn{3}{|c|}{$\begin{array}{c}\text { Febrero del } 2009 \text { a mayo del } 2013 \\
\text { Formación bruta de capital y préstamos de la } \\
\text { banca comercial }\end{array}$} \\
\hline$H_{0}$ & $\lambda_{\text {traza }}$ & $\lambda_{\text {mínimo }}$ & $H_{0}$ & $\lambda_{\text {traza }}$ & $\lambda_{\text {mínimo }}$ \\
\hline$r=0$ & 26.2873 & 15.41 & $r=0$ & 18.1697 & 15.41 \\
\hline$r=1$ & $0.2352^{*}$ & 3.76 & $r=1$ & $0.0085^{*}$ & 3.76 \\
\hline
\end{tabular}

* Significación al 5\%. 
Cuadro 7. Pruebas de cointegración (tercera parte)

\begin{tabular}{|c|c|c|c|c|c|}
\hline \multicolumn{6}{|c|}{ Pruebas de la traza ( $\lambda_{\text {traza }}$ ) y el valor característico mínimo ( $\left.\lambda_{\text {mínimo }}\right)$} \\
\hline \multicolumn{3}{|c|}{$\begin{array}{l}\text { Enero de } 1996 \text { a octubre de } 2008 \\
\text { Actividad industrial e IPC }\end{array}$} & \multicolumn{3}{|c|}{$\begin{array}{l}\text { Febrero del } 2009 \text { a mayo de } 2013 \\
\text { Actividad industrial e IPC }\end{array}$} \\
\hline$H_{0}$ & $\lambda_{\text {traza }}$ & $\lambda_{\text {mínimo }}$ & $H_{0}$ & $\lambda_{\text {traza }}$ & $\lambda_{\text {mínimo }}$ \\
\hline$r=0$ & $9.8909 * *$ & 15.41 & $r=0$ & 21.7424 & 15.41 \\
\hline$r=1$ & 0.4787 & 3.76 & $r=1$ & 5.2668 & 3.76 \\
\hline \multicolumn{3}{|c|}{$\begin{array}{l}\text { Enero de } 1996 \text { a octubre del } 2008 \\
\text { Actividad industrial y préstamos de la banca } \\
\text { comercial }\end{array}$} & \multicolumn{3}{|c|}{$\begin{array}{c}\text { Febrero del } 2009 \text { a mayo del } 2013 \\
\text { Actividad industrial y préstamos de la banca } \\
\text { comercial }\end{array}$} \\
\hline$H_{0}$ & $\lambda_{\text {traza }}$ & $\lambda_{\text {mínimo }}$ & $H_{0}$ & $\lambda_{\text {traza }}$ & $\lambda_{\text {mínimo }}$ \\
\hline$r=0$ & 29.9963 & 15.41 & $r=0$ & $12.8180 *$ & 15.41 \\
\hline$r=1$ & $0.4247^{*}$ & 3.76 & $r=1$ & 0.1282 & 3.76 \\
\hline
\end{tabular}

* Significancia al 5\%.

Los resultados presentados en los cuadros 5, 6 y 7 indican que para el periodo de enero de 1996 a octubre de 2008, tanto el IGAE, como la formación bruta de capital fijo en maquinaria y equipo y la actividad industrial están cointegrados con los préstamos de la banca comercial, resultado que se mantiene para el periodo de febrero del 2009 a mayo del 2013. Asimismo, la formación bruta de capital fijo en maquinaria y equipo y el IPC están cointegrados en el periodo enero de 1996 a octubre de 2008.

\section{CONCLUSIONES}

La crisis financiera de 2008-2009 llamó nuevamente la atención sobre el rol del sector financiero y su vinculación con la economía real. Algunos estudios buscan identificar si el dicho sector incide positivamente en el crecimiento económico, mientras que otras investigaciones analizan la relación de largo plazo que guarda con la actividad económica real. Este estudio se propuso probar si se cumple o no la hipótesis de la desvinculación en el caso de México, por lo que a través de la técnica de cointegración se analiza si el sector financiero y la economía real mantienen una relación de largo plazo, encontrando evidencia positiva de tal re- 
lación entre las variables representativas de la economía real y los préstamos del sector bancario. La evidencia también indica vinculación, antes de la crisis bursátil mundial de octubre de 2008, entre la economía real, ponderada a través de la formación bruta de capital fijo en maquinaria y equipo, y el sector financiero, específicamente el sector bursátil, representado por el IPC.

La diferencia de este estudio con otros realizados sobre México es que se incorpora la posibilidad de cambios estructurales, ya que si estas rupturas no se toman en cuenta, las pruebas tradicionales de cointegración tienden a no rechazar la hipótesis nula (no cointegración), incluso a pesar de que las variables sí se cointegren. Pero en contraste con los resultados de Zavaleta y Urbina (2011), no se encontró evidencia de una relación de largo plazo entre el IGAE y el IPC, antes o después de la crisis financiera de 2008. Una limitante, es que el presente trabajo no incorpora variables que identifiquen al sector externo, ya que no es del interés de los autores, sin embargo, se abre la posibilidad para que futuras investigaciones analicen la vinculación del sector real de la economía o del financiero con el exterior.

\section{REFERENCIAS BIBLIOGRÁFICAS}

Ansotegui, Carmen, y Esteban, María (1997), "Relaciones entre precios y fundamentos en el mercado de capitales español”, Revista de Economía Aplicada, 5(14), pp. 99-117.

Arestis, Philip; Demetriades, Panicos, y Luintel, Kul (2001), "Financial Development and Economic Growth: The Role of Stock Markets", The Journal of Money, Credit and Banking, 33(1), pp. 17-41.

Cagli, Efe; Halac, Umut, y Taskin, Dilvin (2010), "Testing long run relationships between stock market returns and macroeconomic performance: evidence from Turkey", International Research Journal of Finance and Economics, 48, pp. 49-60.

Cheung, Yin-Wong, y Ng, Lilian (1998), "International evidence on the stock market and aggregate economic activity”, Journal of Empirical Finance, 5 (3), pp. 281-296.

Dickey, David, y Fuller, Wayne (1979), "Distribution of the Estimators for Autoregressive Time Series with a Unit Root", Journal of the American Statistical Association, 74 (4), pp. 427-431.

Domingo, Carlos, y Tonella, Giorgio (2000), "Towards a theory of structural change", Structural Change and Economic Dynamics, 11 (1-2), pp. 209-225.

Engle, Robert, y Granger, Clive (1987), "Co-integration and Error correction: Representation, estimation and testing”, Econometrica, 55 (2), pp. 251-276. 
Favara, Giovanni (2003), “An empirical reassessment of the relationship between finance and growth", IMF Working Paper 03/123.

Guiso, Luigi; Sapienza, Paola, y Zingales, Luigi (2004), “Does Local Financial Development Matter?", Quarterly Journal of Economics, 119 (3), pp. 929-69.

Gurley, John, y Shaw, Edward (1955), "Financial aspects of economic development", The American Economic Review, 45 (4), pp. 515-538.

Humpe, Andreas, y Macmillan, Peter (2007), "Can macroeconomic variables explain long term stock market movements? A comparison of the us and Japan", working paper, Centre for Dynamic Macroeconomic Analysis, University of St. Andrews, 07/20.

INEGI (s.f.a), "Banco de Información Económica", en Instituto Nacional de Estadística y Geografía. Consultado el 1 de abril de 2013, en: http://www.inegi.org.mx/ sistemas/bie/.

_ (s.f.b), "Encuesta Industrial Mensual" en Instituto Nacional de Estadística y Geografia. Consultado el 1 de abril de 2013, en: http://www.inegi.org.mx/inegi/ default.aspx?s=est\&c=10554.

_ (s.f.c), "Estructura del scian México" [pdf]. Consultado el 1 de abril de 2013, en: http://www.inegi.gob.mx/est/contenidos/espanol/metodologias/censos/scian/ estructura.pdf.

Johansen, Soren (1988), "Statiscal analysis of cointegration vectors", Journal of Economic Dynamics and Control, 12 (2-3), pp. 231-254.

- (1991), "Estimation and hypothesis testing of cointegration vectors in Gaussan vector autoregressive models", Econometrica, 59 (6), pp. 1551-1580.

- (1992), "Determination of cointegration rank in the presence of a linear trend", Oxford Bulletin of Economics and Statistics, 54 (3), pp. 383-397.

(1995), Likelihood based Inferencein Cointegrated Vector Autoregressive Models, Oxford, Clarendon Press.

Karamustafa, Osman, y Kucukkale, Yakup (2003), "Long run relationships between stock market returns and macroeconomic performance: evidence from Turkey", The Economics Working Paper Archive 0309010.

Kasa, Kenneth (1992), "Common stochastic trends in international stocks markets", Journal of Monetary Economics, 29 (1), pp. 95-124.

King, Robert, y Levine, Ross (1993), "Financial intermediation and economic development", en Colin Mayer and Xavier Vives (eds.), Capital Markets and Financial Intermediation, Cambride, Cambridge University Press, pp. 156-196.

Kwon, Chung, y Shin, Tai (1999), "Cointegration and causality between macroeconomics variables and stock markets returns", Global Finance Journal, 10 (1), pp. 71-81. 
Levine, Ross (1997), "Financial Development and Economic Growth: Views and Agenda", Journal of Economic Literature, 35 (2), pp. 688-726.

Levine, Ross; Loayza, Norman, y Beck, Thorsten (2000), "Financial intermediation and growth: Causality and causes", Journal of Monetary Economics, 46 (1), pp. 31-77.

Levine, Ross, y Zervos, Sara (1996), “Stock Market Development and Long-Run Growth", World Bank Economic Review, 10 (2), pp. 323-39.

Maysami, Ramin; Howe, Lee, y Hamzah, Mohamad (2004), "Relationship between Macroeconomic Variables and Stock Market Indices: Cointegration Evidence from Stock Exchange of Singapore's All-s Sector Indices, Journal Pengurusan, 24 (2004), pp. 47-77.

McMillan, David (2001), “Cointegration Relationships between Stock Market Indices and Economic Activity: Evidence from us Data”, discussion paper 104, Centre for Research into Industry, Enterprise and the Firm, University of St. Andrews.

- (2005), "Time variation in the cointegrating relationship between stock prices and economic activity", International Review of Applied Economics, 19 (3), pp. 359-368.

Menkhoff, Lucas, y Tolksdorf, Norbert (2001), Financial market drift. Decoupling of the financial sector from the real economy?, Germany, Springer-Verlag.

Morales, Andrés, y Rendón, Raúl (1997), "La bolsa mexicana de valores ¿realidad económica o especulación?”, Política y Cultura, 0 (8), pp. 393-410.

Nasseh, Alireza, y Strauss, Jack (2000), "Stock Prices and Domestic and international macroeconomic activity: A cointegration Approach", The Quarterly Review of Economics and Finance, 40 (2), pp. 229-245.

Phillips, Peter, y Perron, Pierre (1988), "Testing for a Unit Root in Time Series Regression", Biometrika, 75 (2), pp. 335-346.

Shen, Chung-Hua, y Lee, Chien-Chiang (2006), "Same Financial Development yet Different Economic Growth: Why?”, Journal of Money, Credit and Banking, 38 (7), pp. 1907-1944.

Solow, Robert (1956), “A Contribution to the Theory of Economic Growth", Quarterly Journal of Economics, 70 (1), pp. 65-94.

Taboada, Eunice, y Sámano, Miguel (2003), “Análisis de cointegración entre el sistema financiero y la economía real en México”, Análisis Económico, XVIII (39), pp. 142-166.

Zavaleta, Osmar, y Urbina, Héctor (2011), "Mercados financieros y desarrollo económico: un análisis sobre México", Ide@s concyteg, 6 (68), pp. 226-235.

Zivot, Eric, y Andrews, Donald (1992), "Further evidence on the Great Crash, the oilprice shock, and the unit root hypothesis", Journal of Business and Economics Statistics, 10 (4), pp. 251-270. 